\section{Regards sur l'économie allemande}

Bulletin économique du CIRAC

$78 \mid 2006$

Varia

\title{
CDU et SPD à la recherche de nouvelles orientations
}

\section{Henrik Uterwedde}

\section{Q OpenEdition}

1 Journals

\section{Édition électronique}

URL : http://journals.openedition.org/rea/743

DOI : $10.4000 /$ rea. 743

ISBN : 978-2-8218-0852-2

ISSN : 1965-0787

\section{Éditeur}

CIRAC

Édition imprimée

Date de publication : 1 octobre 2006

Pagination : 5-10

ISSN : 1156-8992

\section{Référence électronique}

Henrik Uterwedde, "CDU et SPD à la recherche de nouvelles orientations », Regards sur l'économie allemande [En ligne], 78 | octobre 2006, document 1, mis en ligne le 01 octobre 2008, consulté le 19 avril 2019. URL : http://journals.openedition.org/rea/743 ; DOI : 10.4000/rea.743 


\section{CDU et SPD à la recherche de nouvelles orientations}

\section{Henrik Uterwedde}

\begin{abstract}
Signe des temps : alors qu'ils se sont engagés, à l'issue des élections législatives du 19 septembre 2005, dans la formation d'une grande coalition avec leur adversaire, CDU et SPD ont éprouvé chacun le besoin d'élaborer un nouveau programme fondamental mettant en valeur leur profil et leur identité. Ce processus programmatique engagé ressemble, à première vue, à un exercice thérapeutique pour les militants des deux partis qui ont perdu leurs repères idéologiques dans ce mariage inhabituel 'gauche-droite'. II contraste aussi avec les querelles politiciennes entre les deux partis qui caractérisent actuellement le travail de la grande coalition. Mais il s'agit de véritables débats de fond des deux partis qui cherchent chacun, sur la base d'une certaine vision de la société, à clarifier - et au besoin à adapter - leurs choix politiques dans un monde en mutation, notamment dans le domaine de la politique économique et sociale.
\end{abstract}

II ne faut pas oublier que, avec 580000 et 570000 adhérents, SPD et CDU sont deux grands partis de rassemblement dont l'audience va bien au-delà des notables, des élus et des candidats. Enracinés tous les deux dans le territoire (le SPD à lui seul compte 12500 sections locales) et dans la société civile, comme dans le monde syndical et celui des entreprises, les deux partis sont constamment forcés d'arbitrer entre les différentes sensibilités et les multiples groupes d'intérêt qui les caractérisent ; ainsi, la CDU, réputée proche du patronat, connaît une aile syndicale et sociale, comme le SPD, issu du mouvement ouvrier, a un courant entrepreneurial et libéral. Les courants existants dans les deux partis n'ont rien à voir avec les 'écuries' des présidentiables à la française. Ils sont beaucoup moins personnalisés car ils traduisent la pluralité de leurs racines idéologiques historiques: christianisme social, libéralisme et conservatisme au sein de la CDU, marxisme, socialisme démocratique, social-libéralisme et christianisme au SPD.

Le caractère de Volkspartei (parti de rassemblement) se traduit dans un souci constant de fédérer - et, ce faisant, de dépasser - les différentes positions idéologiques et les groupes d'intérêts présents. Par conséquent, ce qui distingue les deux partis - de centre-gauche et de centre-droite - se manifeste bien plus souvent dans les nuances que dans les choix fondamentaux. De même, les débats de fond sur les choix de société, s'ils semblent opposer SPD et CDU dans l'espace public, sont d'abord menés à l'intérieur de chaque parti.

Mais il y a plus : s'ils relancent chacun aujourd'hui un vaste débat sur leurs valeurs et leurs objectifs fondamentaux, c'est que CDU et SPD éprouvent le besoin d'avoir des repères au-delà de la pratique gouvernementale quotidienne, et qu'ils ont le sentiment que, face à un monde en mutation rapide, ces repères doivent être ajustés, voire repensés.

Comme le définit le papier d'orientation du SPD en date du 24 avril : «Le premier programme du SPD au $21^{e}$ siècle doit donner de nouvelles réponses aux défis de l'européanisation, de la mondialisation et des mutations sociales, démographiques et technologiques. Nous vivons dans une période d'évolutions asynchrones. Le nouveau prend forme, et le vieux résiste tout en perdant de
Deux partis de rassemblement que seules distinguent des nuances

Les mutations forcent l'adaptation des programmes politiques 
Ils seront votés à l'automne 2007

a CDU a lancé le débat en février $2006 .$.

... pour répondre à son recul dans l'opinion...

... et à la dilution de son projet de réformes dans la coalition

Courants libéral et social s'affrontent son importance ». A cela s'ajoute le sentiment que l'action gouvernementale récente, y compris celle de la grande coalition, n'a pas toujours été à la hauteur des défis.

La période actuelle, marquée par la perte des certitudes, par la mise en cause des paradigmes fondamentaux du modèle économique et social, semble propice à une réflexion qui vise à "rétablir un accord sur la société dans laquelle nous voulons vivre ». Voilà donc le débat lancé au SPD - mais aussi à la CDU. II doit aboutir au vote d'un nouveau programme en automne 2007 dans les deux partis. Même si le débat vient seulement de commencer, un regard sur les premières contributions est utile car il permet de prendre la mesure non seulement des convergences et des divergences entre les deux partis, mais aussi de leur aptitude à s'ouvrir aux nouvelles questions et aux nouveaux paradigmes.

\section{CDU : recentrage difficile entre valeurs libérales et sociales}

La CDU a ouvert le débat le 20 février 2006 par un congrès sur les valeurs et a constitué une commission du programme. Après une série d'assises régionales au printemps, un second congrès a réuni, le 22 août, des représentants de la société civile et des partenaires sociaux. Aux assises nationales de novembre 2006, un rapport d'étape sera présenté. Le vote du programme définitif est prévu pour la fin 2007.

Pour la CDU, le débat sur le nouveau programme relève d'une action thérapeutique, sorte d'ersatz de cette discussion franche sur l'échec des législatives de septembre 2005. Car cette discussion pour comprendre comment le parti a pu rater une victoire qui semblait éclatante jusqu'à l'approche du scrutin avait été escamotée par la direction dans la foulée des négociations sur la grande coalition puis la constitution du gouvernement Merkel. Mais elle risque de resurgir face aux difficultés de la grande coalition et la chute de la CDU dans les sondages depuis avril. Les intentions de vote en faveur de la CDU-CSU, après être montées de $34 \%$ à $41 \%$ (31 mars 2006), sont retombées depuis à $34 \%$ (7 septembre 2006 ; sondage Deutschlandtrend Infratest-Dimap/ARD), le plus mauvais score depuis le début 2001. Le SPD, lui, est crédité de $28 \%$ seulement, son plus mauvais score depuis les élections législatives du 19 septembre 2005.

Les enjeux de pouvoir ne sont pas loin ; ce sont surtout les barons du parti, les puissants chefs de gouvernement des Länder comme Roland Koch (Hesse), Christian Wulff (Basse-Saxe) ou Jürgen Rüttgers (Rhénanie du Nord-Westphalie) qui tiennent à marquer leur terrain face à la chancelière. Mais le malaise se nourrit plus encore de la frustration d'un parti qui avait lancé une campagne très libérale proposant au pays des réformes hardies et qui se retrouve à un gouvernement 'noir-rouge' dont l'empreinte social-démocrate paraît prédominante aux yeux de certains.

D'où la guerre de positionnement entre les courants libéral et social au sein de la CDU. Le premier, représenté par exemple par le Wirtschaftsrat, association patronale proche du parti (www.wirtschaftsrat.de), avait beaucoup misé sur la promesse de réformes libérales et l'alliance avec le parti libéral (FDP) ; il craint une " social-démocratisation rampante » de la CDU, et il critique l'alliance tacite entre les tenants de la politique sociale dans les deux partis, qui dominerait la grande coalition au détriment des besoins de l'économie.

A cela, le courant représentant les salariés, la Christlich-demokratische Arbeitnehmerschaft (CDA, www.cda-bund.de), rétorque que l'élection au Bundestag a été perdue parce que la campagne libérale de la CDU se serait trop éloignée du peuple et aurait suscité des angoisses dans la population. D'où la revendication d'un recentrage des positions, réhabilitant la justice sociale en tant que valeur fondamentale du parti. Le fait que le ministre-président Rüttgers se prononce, en août 2006, contre «l'illusion » de croire que la baisse des impôts et des sa- 
laires créerait automatiquement des emplois, credo des libéraux et ligne du parti jusqu'à aujourd'hui, est révélateur du nouveau débat et des controverses qu'il suscite.

Voilà donc la difficile tâche qu'affronte la direction de la CDU : comment arriver à une synthèse productive entre les positions d'un parti qui reste caractérisé par la pluralité de ses racines, chrétiennes-sociales, libérales et conservatrices ?

En attendant un premier texte d'orientation - pour l'instant, la direction s'est limitée prudemment à lancer une batterie de questions - on peut déceler les contours suivants dans le domaine économique et social :

En ce qui concerne les valeurs fondamentales, la liberté prend une place particulière. D'ailleurs, le congrès sur les valeurs était placé sous le titre "davantage de justice sociale par davantage de liberté ». Un accent particulier est mis sur le principe de concurrence et de compétition comme éléments de régulation, pas seulement en économie : compétition des systèmes d'éducation, des universités, fédéralisme compétitif... La justice sociale est vue d'abord comme équité des chances ou justice intergénérationnelle, qui admet des inégalités dans les résultats.

La question de l'Etat - qui se confond en Allemagne, rappelons-le, avec celle de tous les acteurs public, du niveau fédéral au local en passant par celui des Länder - est un autre point de distinction entre CDU et SPD. Tout en se démarquant d'une vision paléolibérale (Nachtwächterstaat : "Etat gendarme »), les chrétiens-démocrates insistent sur les insuffisances de l'Etat devenu trop interventionniste dans trop de domaines. Selon les termes de Ronald Pofalla, secrétaire général de la CDU, l'Etat devra " se retirer de certaines tâches afin de pouvoir réaliser d'autres objectifs de manière plus efficace " (Frankfurter Allgemeine Zeitung, 24-04-06). La CDU tend vers un redéploiement des activités de l'Etat vers les domaines qui commandent l'économie du savoir (enseignement, formation, innovation, recherche) ou la cohésion nationale (intégration culturelle et sociale des immigrés). En outre, les principes de la subsidiarité, de la responsabilité individuelle et de la "société citoyenne " (Bürgergesellschaft) sont rappelés pour inviter les citoyens à prendre en charge eux-mêmes, individuellement et collectivement, des tâches que l'intervention publique ne peut pas, ou ne peut plus, assurer.

L'économie sociale de marché (Soziale Marktwirtschaft, en réalité : économie de marché sociale), notion sur laquelle la CDU réclame à juste titre une sorte de brevet puisqu'elle fut inventée par Ludwig Erhard, est vue comme un modèle de société dépassant la seule sphère économique. Ce modèle est placé devant un double défi de renouvellement: l'adaptation aux conditions d'une économie globale, et sa légitimité dans la population. Or c'est là, sur ce dernier point, que le bât blesse, comme l'a rappelé l'échec relatif de la campagne électorale de 2005. Angela Merkel a clairement mis le doigt sur le fait que pour beaucoup d'Allemands, et pas seulement à l'est, l'économie sociale de marché n'est plus considérée comme une garantie de justice sociale et de protection. Dans son discours lors du congrès sur les valeurs de la CDU, elle s'était exprimée ainsi : " II y a beaucoup de questions, et il y a aussi des angoisses. Nous ne pouvons pas écarter ces angoisses qui naissent de la mondialisation. Nous ne pouvons pas dire simplement: c'est comme ça dans un monde globalisé. Nous devons au contraire prendre au sérieux ces angoisses, et nous devons également faire apparaître et mettre à profit les chances de la mondialisation ».

Quant aux aspects internationaux, la CDU se prononce pour l'exportation du modèle d'économie sociale de marché et pour l'établissement d'un cadre international de régulation (Internationale Soziale Marktwirtschaft).

\section{SPD : Renouveler le modèle allemand pour mieux le sauver}

Les débats sur un nouveau programme fondamental du SPD remontent à 2000 et ont été accélérés au début de cette année. En avril 2006, un papier d'orientation a été présenté, qui a été suivi d'une phase de discussion tant interne qu'externe (voir www.programmdebatte.spd.de). En janvier 2007, la direction doit présenter une proposition de programme ; après une deuxième phase de discussion, il doit être voté à l'automne 2007.

Pour le SPD, qui a perdu de justesse les élections au Bundestag mais sauvé la face en s'imposant à la CDU comme partenaire de gouvernement incontournable et ex aequo (voir REA 75/05), le débat sur un programme fondamental
Ils doivent être (ré)conciliés...

... autour de valeurs communes renouvelées :

- seule la liberté permet la justice sociale

- la responsabilité individuelle prime sur le rôle de l'Etat

- fidélité au modèle de l'économie de marché sociale

- le marché mondial requiert une approche ordo-libérale

Le programme du SPD en débat depuis 2000

Le SPD a perdu ses repères sous les gouvernements Schröder 
Concilier programme 'de gauche' et responsabilité gouvernementale

Un positionnement prudemment moderniste :...

- Un Etat « actif et coopératif »...

- un Etat social « de prévoyance »...

- une réforme graduelle du capitalisme rhénan, sans rupture... est l'occasion de redonner des repères programmatiques à un parti qui en a bien besoin : traditionnellement, le SPD est davantage que la CDU un parti de programmes qui veut définir les finalités du travail gouvernemental quand il exerce le pouvoir. Ces repères avaient été perdus au fil de l'action du gouvernement Schröder qui, pendant sept ans, a d'abord semblé hésiter entre une politique traditionnaliste et des réformes prudentes, avant de lancer brusquement et sans débat préalable une politique de réformes tous azimuts connue sous le nom d'Agenda 2010 (voir REA 75/05). Courageuse mais mal préparée et mal expliquée, cette politique a provoqué le désarroi au sein du parti, la naissance d'un parti de la gauche socialiste menée entre autres par son ancien leader Oskar Lafontaine et, enfin, la défaite de Gerhard Schröder aux élections de 2005. Cela dit, c'est moins le rejet de la politique de réformes de Gerhard Schröder que son incapacité à lui donner un sens qui a semé le désarroi dans le parti.

Comment s'affirmer comme un parti de gouvernement, responsable et capable de renouveler le pays tout en marquant le terrain de la gauche ? Comment éviter à la fois la dérive gestionnaire et celle d'un repli stérile sur des positions traditionnelles? Tels sont les défis du débat social-démocrate. Comme à la CDU, les tensions entre les différents courants de pensée se manifestent ainsi, surtout entre l'aile syndicale (Arbeitsgemeinschaft für Arbeitnehmerfragen; voir afa.spd.de) et l'aile moderniste et sociale-libérale ; cette dernière s'exprime notamment dans le périodique Berliner Republik, l'organe du Netzwerk, un réseau de jeunes députés modernistes (www.b-republik.de).

Dans le texte d'orientation comme dans les premières prises de position se dégage dès lors un positionnement prudemment moderniste.

Ainsi, comme à la CDU, les principes de la Bürgergesellschaft, de la responsabilité individuelle et de la subsidiarité sont mis en avant. Cela dit, le rôle d'un "Etat actif et coopératif » est défini de manière plus offensive, et l'idée d'un "Etat modeste » est rejetée. Mais l'ouverture à une redéfinition des tâches et au redéploiement des ressources publiques est claire. Ainsi, les propositions de Peer Steinbrück, ministre fédéral des Finances et moderniste du SPD, sur le rôle de l'État sont assez proches des positions des chrétiens-démocrates. Dans un discours tenu à la Fondation Friedrich Ebert en juin dernier, il propose un équilibre entre l'Etat, le marché et la société civile pour assurer les tâches publiques ; si l'Etat reste indispensable, "certaines tâches peuvent être résolues de manière plus efficace par le marché et d'autres ne peuvent être prises en charge que par la société civile ". D'où la revendication d'une concentration de l'action et des ressources publiques sur des domaines essentiels comme l'enseignement, la recherche, l'innovation, la formation ou la politique familiale. Mais il est vrai que, dans son ensemble, le SPD reste plus confiant dans l'intervention de l'Etat que la CDU, comme en témoigne la revendication de recettes publiques suffisantes pour financer les activités publiques, et aussi la défense d'un secteur de biens publics non soumis aux lois du marché.

Pour le SPD, la défense de la protection sociale passe par son renouvellement. C'est pourquoi le paradigme d'un "Etat social de prévoyance " (vorsorgender Sozialstaat) est mis en avant, qui prendrait mieux en charge les nouveaux problèmes sociaux (précarité, pauvreté), investirait davantage dans la prévention et dans les infrastructures sociales mais ferait aussi appel à la responsabilité individuelle, pour assurer une meilleure balance entre droits et devoirs dans la citoyenneté sociale.

Dans tous les textes apparaît un attachement au capitalisme coopératif du modèle rhénan. Ainsi, le texte d'orientation se prononce en faveur d'alliances entre les entreprises, la recherche, les salariés et les acteurs politiques ; il favorise une stratégie de la compétitivité fondée sur la qualité et non sur les bas salaires; il demande une protection des " entreprises performantes " contre des OPA inamicales ; il est attaché à une économie qui favorise la logique industrielle contre la dictature financière et préfère le long terme au court-termisme, selon la belle formule du président du SPD, Kurt Beck : " une culture d'entreprise qui pense à la prochaine génération et pas seulement au prochain trimestre » (discours du 24 avril). Aussi, si changement il doit y avoir, c'est un gradualisme respectant les traditions sociales du pays qui est proposé, et toute stratégie de rupture est rejetée. 
La maîtrise de la mondialisation figure parmi les priorités du SPD. De manière encore plus accentuée que la CDU, le parti préconise un cadre international de régulation des marchés, qui fixerait des règles à la finance internationale et des standards sociaux et écologiques dans l'intérêt d'un commerce mondial équitable. De même, l'Union européenne est destinée à devenir un acteur économique important. Elle devrait combattre le dumping fiscal et social par une politique des standards sociaux et par la coordination des politiques fiscales et sociales. Enfin, la coordination des politiques économiques, financières et monétaires devrait être renforcée. Bien sûr, la stratégie d'innovation de Lisbonne ne manque pas ; elle incarne l'appel au renouvellement du modèle social européen.

\section{Consensus}

Tout d'abord, on est frappé par le grand nombre d'éléments visibles de consensus - ce qui, rappelons-le, n'est pas un mot tabou outre-Rhin, même entre les adversaires politiques que sont le SPD et la CDU. La culture de gouvernement qu'ils incarnent tous les deux, forts de leur expérience quasi permanente d'exercice du pouvoir au niveau fédéral comme à celui des Länder, leur interdit toute polarisation excessive. Depuis longtemps, les deux partis partagent les valeurs fondamentales qui doivent orienter leur politique : liberté, justice, solidarité. Comme le formulait Angela Merkel dans son discours sur les valeurs : "II est intéressant de relever qu'en Allemagne il n'y a pas de débat sur le fait que la liberté, la solidarité et la justice constituent nos valeurs fondamentales. Ceci nous unit par exemple avec l'autre grand parti populaire ". Ce qui fait débat, en revanche, c'est la configuration entre ces trois valeurs - leur poids respectif, la résolution des conflits potentiels entre elles, et ainsi de suite, d'où l'existence de lectures différentes des valeurs partagés.

Même consensus relatif : l'attachement à l'économie sociale de marché (Soziale Marktwirtschaft), doctrine élaboréé par l'ordolibéralisme allemand dans les années 1940 et popularisée par Ludwig Erhard. Depuis l'alignement du SPD dans son programme de Bad Godesberg en 1959, SPD et CDU partagent en principe l'idée d'un modèle de capitalisme social, qui cherche un équilibre entre les lois du marché et l'objectif de justice sociale, en rejetant tout modèle ultralibéral. Cette convergence fondamentale entre les deux grands partis est le fondement même du capitalisme social allemand, qui a besoin d'être soutenu par l'ensemble des acteurs économiques et sociaux, voire des citoyens.

Là encore, il est clair qu'il y a des lectures fortement différentes pour définir la pratique de l'économie sociale de marché. Mais il est intéressant de noter que, alors que le SPD au pouvoir a fait des pas vers une redéfinition partielle du « social » dans l'économie sociale de marché, la CDU semble tirer les conséquences de l'échec relatif de sa stratégie de rupture libérale proposée lors de la campagne électorale, sous l'impulsion du parti libéral FDP, mais aussi de son aile patronale. En témoigne l'aveu d'Angela Merkel lors du congrès sur les valeurs : "L'adhésion des Allemands à l'économie sociale de marché reposait sur la conviction intime que l'économie sociale de marché est synonyme de justice sociale. Mais ce consensus sur le fondement de notre modèle a perdu de sa consistance, et il nous faut bien reconnaître que, aujourd'hui, seule une minorité partage encore ce point de vue... Et je suis persuadée que le concept d'économie sociale de marché ne pourra pas survivre si nous ne parvenons pas à rétablir dans l'opinion ce profond sentiment de justice sociale ».

Rien d'étonnant à cette convergence relative entre deux partis de rassemblement qui représentent, chacun à sa manière et dans des configurations différentes, la pluralité des intérêts sociaux et des courants de pensée. Cela les amène à favoriser des approches gradualistes et à exclure des solutions simplistes ou des stratégies de rupture. Cette démarche correspond tout à fait à la culture politique allemande centriste qui a horreur des extrêmes, au caractère coopératif du capitalisme rhénan, à un système politique multi-acteurs qui exige
- la Stratégie de Lisbonne est assumée au nom d'un modèle social européen

Des valeurs partagées : liberté, justice, solidarité

Attachement partagé à l'économie de marché sociale

Des partis de rassemblement, reflets d'un système politique multi-acteurs 
en permanence des compromis et à une société pluraliste et complexe. C'est pourquoi la critique récurrente de la «lenteur » et de la "timidité » des réformes en Allemagne passe à côté de l'essentiel car elle méconnaît ces données politiques et sociétales fondamentales qui déterminent la politique allemande.

Une rhétorique de la « rupture » telle qu'elle surgit de nouveau en France à l'approche de l'élection présidentielle ne saurait pas convaincre les électeurs allemands. A titre d'exemple, le public a ainsi pu applaudir le député CDU Friedrich Merz, qui proposait en 2005 de simplifier radicalement le système fiscal allemand - au point que la déclaration annuelle d'impôt tiendrait sur un... dessous de bock. Mais au moment de la présentation du projet de concrétisation, le même public a soudain découvert ses intérêts multiples et les mille raisons interdisant la réalisation du projet. Le rejet de l'esquisse de réforme fiscale élaborée en 2003 par Paul Kirchhoff et mise en débat lors de la campagne électorale s'explique de la même manière.

C'est dire que les textes d'orientation qui guident les débats programmatiques au SPD et à la CDU sont forcément des papiers de compromis, bien pondérés et où il est parfois difficile de déceler des idées innovatrices.

Cela dit, les Paradigmes du renouveau de la protection sociale, du redéploiement de l'action publique et de la société citoyenne, qui se dégagent plus ou moins clairement dans les deux partis, témoignent d'une prise de conscience collective du fait qu'on ne peut pas tout demander à l'Etat, et que la redéfinition partielle des domaines de l'Etat, du marché et de la société civile, sans être une rupture, est à l'ordre du jour. Même si le chemin semble encore long jusqu'à une acceptation générale de ces paradigmes dans la société allemande, et si l'on peut gloser sur le décalage entre le dire et le faire en regardant le travail actuel de la grande coalition, les débats programmatiques engagés par la CDU et le SPD sont des préalables nécessaires à l'élaboration de nouveaux compromis institutionnalisés au sein du capitalisme allemand. Car la politique du renouvellement engagée ne sera pas durable si ses orientations fondamentales ne sont pas soutenues par les citoyens.

\section{Indications bibliographiques :}

BECK K., « Kraft der Erneuerung - Auf dem Weg zu einem neuen Grundsatzprogramm », discours au congrès de Berlin, 24 avril 2006 (www.programmdebatte.spd.de)

BouRgeols I., "Années Schröder: les 'recentrages' successifs de la politique économique et sociale, Regards sur l'économie allemande, 75/05

ColletIS G., "Mutation du 'modèle rhénan' et avenir du modèle européen », Regards sur l'économie allemande, 67/04

Débat programmatique de la CDU : www.grundsatzprogramm.cdu.de

Kraft der Erneuerung. Soziale Gerechtigkeit für das 21. Jahrhundert, papier d'orientation, 24 avril 2006. Ce papier et d'autres documents et contributions sont rassemblés sur le site www.programmdebatte.spd.de

StEInBRüCK P., " Die Zukunft der sozialen Demokratie - die Rolle des Staates ", discours à la Fondation Friedrich Ebert, Potsdam, 7 juin 2006 (www.programmdebatte.spd.de)

UTERWEDDE H., "'Capitalisme rhénan' : défis d'adaptation et compétitivité virtuelle ", in BOURGEOIS I. (dir.), Le modèle social allemand en mutation, CIRAC, 2005. 